\title{
Excretion of Nicotinic Acid by Biotin-Deficient Saccharomyces cerevisiae
}

\author{
By A. H. ROSE \\ Microbiology Laboratory, Department of Applied Biochemistry, Heriot-Watt College, \\ Edinburgh, Scotland
}

(Received 12 February 1960)

\section{SUMMARY}

Growth of a strain of Saccharomyces cerevisiae in a glucose salts vitamins medium, containing suboptimal concentrations of biotin, was accompanied by the appearance in the medium of a free form of nicotinic acid, and also a heat-labile combined form of this substance; these were identified chromatographically as nicotinic acid and desamido-DPN (nicotinic acid adenine dinucleotide), respectively. The effect of biotin concentration and incubation time on the excretion of these substances is reported. The presence of adenine in the growth medium caused a marked decrease in the excretion of both forms of nicotinic acid, but hypoxanthine and 4-amino5-imidazole carboxamide had no observable effect. Addition of DL-aspartic acid to the medium also brought about a decrease in the amounts excreted, although concentrations of this amino acid in excess of $0.5 \times 10^{-3} \mathrm{M}$ stimulated excretion of desamido-DPN. It is concluded that excretion of free nicotinic and desamido-DPN is largely the result of an impairment in the ability of the biotin-deficient yeast to produce adenine, which in turn leads to a derangement in the synthesis of pyridine nucleotides.

\section{INTRODUCTION}

Several workers have studied the growth of biotin-requiring micro-organisms in media containing suboptimal concentrations of the vitamin, in order to obtain an insight into the role of biotin in the metabolism of living cells. Micro-organisms grown under these conditions have been shown to lose the ability to carry out certain metabolic reactions, principally those involving carboxylation (Lardy, Potter \& Elvehjem, 1947; Shive \& Rogers, 1947; Lichstein \& Umbreit, 1947) and transamination (Lichstein \& Christman, 1948). Biotin has also been shown to be essential for hexokinase activity in Saccharomyces cerevisiae (Strauss \& Moat, 1958) and for succinic acid dehydrogenase activity in Escherichia coli (Ajl, Hart \& Werkman, 1950); in Streptococcus lactis it is associated with ability to convert ornithine into citrulline (Ravel, Grona, Humphreys \& Shive, 1959). There is evidence too of a relationship between biotin and fatty acid synthesis in lactic acid bacteria, although the nature of the relationship has not been elucidated (Hofmann, O'Leary, Yoho \& Liu, 1959). Several studies have also been made on the accumulation of biosynthetic intermediates in the culture medium during growth of micro-organisms under conditions of biotin deficiency. Among the intermediates which have been detected are an aromatic amine, probably 5 -amino-imidazole riboside (Chamberlain, Cutts \& Rainbow, 1952; Lones, Rainbow \& Woodward, 1958; Moat, Wilkins \& 
Friedman, 1956) and hypoxanthine (Chamberlain \& Rainbow, 1954), excretion of both these compounds being the result of an impairment in the ability of biotindeficient micro-organisms to synthesize purines. It has also been reported that under conditions of biotin deficiency, pyruvate, dimethyl-pyruvate and $\alpha$-ketoglutarate are excreted by Piricularia oryzae (Katsuki, 1959a), while pyruvate can also be detected in filtrates from biotin-deficient cultures of Bacillus macerans (Katsuki, 1959b).

In a previous paper (Rose \& Nickerson, 1956), growth of several biotin-requiring yeasts, in media containing suboptimal concentrations of biotin, was reported to initiate or to enhance greatly the excretion of nicotinic acid by these organisms. However, the factors which affected the excretion were not examined in detail, nor was the form in which the vitamin appeared in the growth medium ascertained. Further studies on excretion of nicotinic acid by a strain of Saccharomyces cerevisiae have recently been carried out in this laboratory, and this paper reports the results obtained, certain of which have already been described in brief (Rose, 1960). In light of these results, it would appear that excretion of nicotinic acid, in both free and combined forms, is largely a reflexion of the inability of the biotin-deficient yeast to synthesize adenine.

\section{METHODS}

\section{Experimental cultures}

Organism. The biotin-requiring strain of Saccharomyces cerevisiae (Fleischmann) used in this study was obtained from the Division of Applied Biology, National Research Council of Canada, Ottawa. It was maintained on slopes of malt wort agar (10\%, (w/v), 'Muntona' spray-dried malt extract (Munton and Fison Ltd., Stowmarket, Suffolk) $+2 \%(w / v)$ agar); the cultures were stored at $2-4^{\circ}$.

Medium. The basal medium ( $\mathrm{pH} 4 \cdot 5$ ) used was that described by Rose \& Nickerson (1956), but with biotin omitted. Various test substances were incorporated in the medium as described later. These substances were screened for possible contamination with biotin by examining their ability to stimulate growth of the biotin-requiring strain of Saccharomyces cerevisiae in biotin-free medium. Substances so contaminated were washed repeatedly with ice-cold water and with $95 \%(v / v)$ ethanol in water until they were shown to be free from detectable amounts of biotin.

Tube tests. Cultures (6 ml.) of the yeast were grown in Samco tubes as described by Northam \& Norris (1951). When large volumes of culture filtrate were required, $100 \mathrm{ml}$. cultures in $350 \mathrm{ml}$. conical flasks were grown. Inocula were prepared by transferring a pin-head of growth from a $48 \mathrm{hr}$. slope culture into $6 \mathrm{ml}$. of biotinfree basal medium and incubating the culture at $25^{\circ}$ for $18 \mathrm{hr}$., during which time the amount of organism in the culture increased from $0 \cdot 09-0 \cdot 11$ to $0 \cdot 32-0.38 \mathrm{mg}$. dry wt. yeast $/ \mathrm{ml}$. Organisms from these cultures were washed three times with $6 \mathrm{ml}$. portions of $\mathrm{M} / 15 \mathrm{KH}_{2} \mathrm{PO}_{4}$, and $0.6 \mathrm{ml}$. of the final suspension (6 ml.) was added to a further $6 \mathrm{ml}$. of buffer. One drop of the diluted suspension was then added to each tube containing $6 \mathrm{ml}$. biotin-free medium; flasks containing $100 \mathrm{ml}$. medium were inoculated with 17 drops of the diluted suspension. This procedure was preferred to that used previously (Rose \& Nickerson, 1956) because lower blank readings were recorded when the yeast was inoculated into biotin-free basal medium. Experimental cultures were incubated statically at $25^{\circ}$. Growth was measured 
turbidimetrically, using the EEL nephelometer (model ' $A$ ') or the Hilger 'Spekker' absorptiometer (model H 760 with neutral green-grey H 508 filters), turbidity readings being related to dry weight of yeast by means of calibration curves.

Culture filtrates were obtained by centrifuging experimental cultures at $2500 \mathrm{rev}$./ min., and decanting the supernatant liquid. The filtrate was adjusted to $\mathbf{p H} 4.5$ with $2 \%(w / v)$ sodium hydroxide, and then sterilized by Seitz filtration ('Carlson' Grade 'E.K.' $3.5 \mathrm{~cm}$. filter pads) the first $2 \mathrm{ml}$. of filtrate being rejected. Seitz filtration did not appear to alter significantly the concentrations of nicotinic acid, nicotinamide or diphosphopyridine nucleotide (DPN) in the filtrates, since excellent recovery values were obtained when standard solutions of these compounds were assayed after passage through the Seitz filter. Sterile culture filtrates were stored at $2-4^{\circ}$ before examination.

\section{Analytical methods}

Nicotinic acid assays. The nicotinic acid contents of culture filtrates were determined microbiologically using a strain of Candida pseudotropicalis (National Collection of Yeast Cultures 143, Brewing Industry Research Foundation, Nutfield, Surrey), and using a modification of the method described by Williams (1946). Portions (6 ml.) of basal medium (Rose \& Nickerson, 1956), containing known amounts of nicotinic acid or of sterile yeast culture filtrate, were autoclaved momentarily at $10 \mathrm{lb}$./sq.in. in Samco tubes. Inocula were prepared as described previously (Rose \& Nickerson, 1956), and the cultures incubated statically at $25^{\circ}$ for $72 \mathrm{hr}$., after which the turbidities of the cultures were measured in the EEL nephelometer. A linear relationship was obtained by plotting response (turbidity reading) against the logarithm of the dose of nicotinic acid; the assay range 20$100 \mathrm{~m} \mu \mathrm{g}$./tube was used in calculating the nicotinic acid contents of culture filtrates. The assay method was found to be reliable, and to give consistently valid results (Bliss, 1956).

Chromatography. Culture filtrates were examined chromatographically on Whatman no. 1 paper, using a solvent consisting of a mixture of 7 parts of $95 \%(v / v)$ ethanol in water and 3 parts $\mathrm{M}$ ammonium acetate, adjusted to $\mathrm{pH} \mathrm{5.0}$ with $\mathrm{HCl}$ (Preiss \& Handler, 1958a). Filtrates or extracts from filtrates were applied as spots (c. $3 \mu \mathrm{l}$.), larger amounts being applied by successive application of $3 \mu \mathrm{l}$. spots. Papers were irrigated for 16-20 hr. at room temperature and, when the solvent front had descended $40-42 \mathrm{~cm}$., were dried with a hair dryer. Chromatogram papers were examined under ultraviolet (u.v.) radiation (Hanovia 'Chromatolite'), nicotinic acid, nicotinamide, and pyridine nucleotides then appearing as quench spots. Nicotinamide nucleotides were distinguished from the nicotinic acid analogues by placing the chromatogram paper in a tank containing a mixture of equal volumes of methyl ethyl ketone and conc. ammonia (sp.gr. 0.88) for $1 \mathrm{hr}$., after which only nicotinamide derivatives appeared as fluorescent spots under u.v. irradiation (Kodicek \& Reddi, 1951). Spots of nicotinic acid and nicotinamide were further distinguished by placing the chromatogram paper in a tank over cyanogen bromide (Wang \& Kodicek, 1943) for $1 \mathrm{hr}$., and then spraying with $2 \%(w / v) p$-aminobenzoic acid; spots of nicotinic acid then appeared yellow and those of nicotinamide orange-yellow (Kodicek \& Reddi, 1951). 


\section{RESULTS}

\section{Influence of biotin concentration and incubation time on excretion of nicotinic acid}

In a series of preliminary experiments, cultures of the yeast grown in basal media supplemented with a wide range of biotin concentrations were removed at intervals, and the culture filtrates analysed for nicotinic acid. When the biotin concentration in the medium was less than $9 \cdot 0 \times 10^{-10} \mathrm{M}$, growth was restricted, but it was not until the concentration was below $5.0 \times 10^{-10} \mathrm{M}$ that nicotinic acid could be detected in the culture filtrate. Nicotinic acid activity reached a maximum in filtrates from 7 -day cultures of the yeast in media containing $0.3-0.5 \times 10^{-10} \mathrm{M}$-biotin. Organisms from these cultures were coloured pink, but in the absence of added methionine, only very small amounts of aromatic amine were detectable in the culture filtrates (Chamberlain \& Rainbow, 1954).

When filtrates from cultures of the yeast grown in media containing $0.4 \times 10^{-10} \mathrm{M}-$ biotin were assayed for nicotinic acid activity by adding portions of sterile filtrate aseptically to assay medium, instead of autoclaving the sample with medium, nicotinic acid potency appeared to be lower. An examination of the specificity of response of the strain of Candida pseudotropicalis used in the microbiological assay showed that the organism responded identically to nicotinic acid and nicotinamide, but that diphosphopyridine nucleotide (DPN) elicited a detectable growth response from the organism only in relatively high concentrations (greater than $100 \mathrm{~m} \mu \mathrm{g}$. nicotinic acid equivalent $/ \mathrm{ml}$.). It was concluded, therefore, that nicotinic acid was present in culture filtrates in a free assayable form (possibly nicotinic acid or nicotinamide), and as one or more heat-labile combined forms, which were broken down to assayable forms of the vitamin when filtrates were autoclaved momentarily at $10 \mathrm{lb}$./sq.in. with basal medium. Autoclaving for longer periods did not lead to any further increase in the nicotinic acid potency of filtrates.

Filtrates from cultures of the yeast grown in basal media containing different concentrations of biotin were therefore assayed for total nicotinic acid by using the standard assay procedure, and also for free nicotinic acid by adding portions aseptically to assay medium; the content of heat-labile, combined nicotinic acid was obtained by difference. The data in Fig. 1 show the effect of biotin concentration on growth and excretion of free and combined nicotinic acid by the yeast after incubation for $170 \mathrm{hr}$. In filtrates from media containing $0.3-0.5 \times 10^{-10} \mathrm{M}$-biotin, in which total nicotinic acid activity was greatest, there were approximately equal amounts of free and combined vitamin; this proportion remained roughly constant as the total amount of nicotinic acid excreted decreased with increasing biotin concentration. Nicotinic acid was not detected in filtrates from media containing more than $5 \cdot 0 \times 10^{-10} \mathrm{M}$-biotin. The small amount of nicotinic acid excreted in biotinfree medium was predominantly in the free form. As shown in Fig. 2, the concentrations of free and combined nicotinic acid in filtrates from cultures of the yeast grown in media containing $0.4 \times 10^{-10} \mathrm{M}$-biotin increased steadily with time, reaching a maximum after about $170 \mathrm{hr}$. of incubation. Thereafter, the concentration of free nicotinic acid continued to increase, while that of the combined form decreased sharply, possibly as a result of its breakdown by enzymes liberated from the yeast cells. 


\section{Characterization of the free and combined forms of nicotinic acid}

When filtrates from cultures of the yeast grown in media containing $0.4 \times 10^{-10} \mathrm{M}$ biotin were examined chromatographically, scanning the chromatograms under u.v. irradiation revealed the presence in these filtrates of free nicotinic acid but not nicotinamide. These findings were corroborated when the papers were treated with cyanogen bromide and then sprayed with $p$-aminobenzoic acid. Similar results were obtained on examining other yeast culture filtrates which had been shown by microbiological assay to contain the free form of the vitamin.

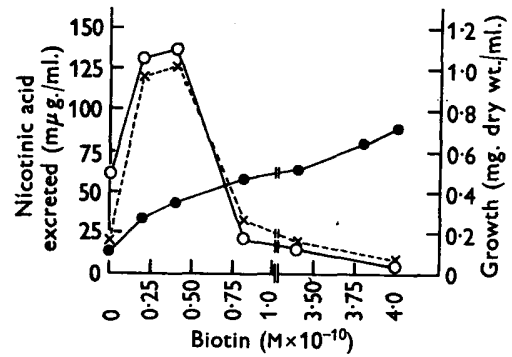

Fig. 1

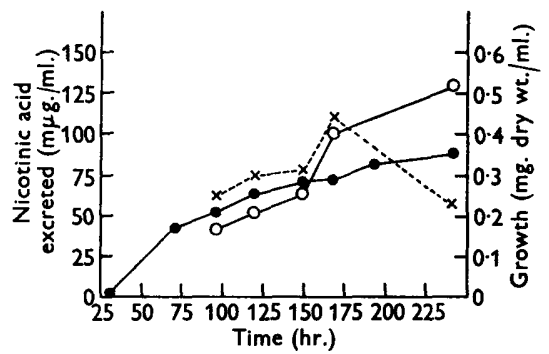

Fig. 2

Fig. 1. Effect of biotin concentration on growth (- - ) and excretion of free $(0-0)$ and combined $(x---x)$ nicotinic acid by the yeast after incubation for $170 \mathrm{hr}$.

Fig. 2. Effect of incubation time on growth $(-\longrightarrow)$ and excretion of free $(\mathrm{O}-\mathrm{O})$ and combined $(x---x)$ nicotinic acid by the yeast in a medium containing $0.4 \times 10^{-10} \mathrm{M}-$ biotin.

When culture filtrates containing combined nicotinic acid were autoclaved momentarily at $10 \mathrm{lb}$./sq.in., and then examined chromatographically, again only nicotinic acid was detected. Since it is known that nicotinamide is not deaminated under these conditions (Preiss \& Handler, 1958a), it was concluded that the vitamin was present in the combined form as nicotinic acid and not as the amide. The conditions required for liberation of nicotinic acid from the heat-labile combined form closely resembled those required to liberate nicotinic acid from pyridine nucleotides (Preiss \& Handler, 1958a). However, the presence of extraneous u.v.-absorbing material precluded the possibility of examining filtrates chromatographically for the presence of pyridine nucleotides. Mercuric acetate (0.8 g. dissolved in $15 \mathrm{ml}$. water) was therefore added to portions $(100 \mathrm{ml}$.) of filtrate obtained from cultures of the yeast grown in media containing $0.4 \times 10^{-10} \mathrm{M}$-biotin, and the resulting precipitate, which included the mercuric derivatives of any pyridine nucleotides present (Singer \& Kearney, 1954) was allowed to settle overnight at $2-4^{\circ}$. The precipitate was then decomposed by the procedure described by Chamberlain \& Rainbow (1954). The extracts prepared in this way were examined chromatographically, and were shown to contain a substance having an $\boldsymbol{R}_{F}$ value identical with that of desamido-DPN (nicotinic acid adenine dinucleotide) an authentic specimen of which was kindly supplied by Dr N. O. Kaplan (Table 1). This spot did not fluoresce after the chromatogram paper had been treated with methyl ethyl ketone and ammonia (Kodicek \& Reddi, 1951), thus confirming that it was not a nicotinamide nucleotide. No other pyridine nucleotide was detected 
in these extracts, although they did contain hypoxanthine (Chamberlain \& Rainbow, 1954). Control chromatograms were prepared with extracts from basal media supplemented with nicotinic acid, nicotinamide, DPN, desamido-DPN, or desamido-NMN (nicotinic acid mononucleotide) which was obtained by hydrolysis of desamido-DPN (Preiss \& Handler, 1958a). Further proof of the identity of the substance present in the extracts was obtained by hydrolysis of a portion of extract (0.5 ml.) with $0.1 \mathrm{~N}-\mathrm{HCl}$ at $100^{\circ}$ for $10 \mathrm{~min}$. (Preiss \& Handler, 1958a) when chromatographic examination of the hydrolysate revealed the presence of adenine, nicotinic acid, and of a substance having an $\boldsymbol{R}_{F}$ value of $\mathbf{0 \cdot 2 4}$, in addition to some residual desamido-DPN (Table 1). A similar chromatographic pattern was obtained on examination of an extract prepared from basal medium supplemented with acid-hydrolysed desamido-DPN. No pyridine nucleotide was detected in extracts prepared from filtrates which had been autoclaved momentarily at $10 \mathrm{lb} . / \mathrm{sq} . \mathrm{in}$.

Table 1. $R_{\boldsymbol{r}}$ values of u.v.-absorbing substances in supplemented basal media and in extracts prepared from filtrates of cultures of Saccharomyces cerevisiae

\begin{tabular}{|c|c|}
\hline Nature of the spot & $\begin{array}{c}\boldsymbol{R}_{\boldsymbol{F}} \text { value } \\
\text { (average } \\
\text { of three } \\
\text { chromatograms) }\end{array}$ \\
\hline \multicolumn{2}{|l|}{$\begin{array}{l}\text { Reference compounds incorporated in basal } \\
\text { medium }\end{array}$} \\
\hline Nicotinic acid & $0 \cdot 76$ \\
\hline Nicotinamide & 0.79 \\
\hline Nicotinamide mononucleotide (NMN)* & $\mathbf{0 . 3 1}$ \\
\hline Desamido-NMN† & $\mathbf{0 \cdot 2 4}$ \\
\hline Diphosphopyridine nucleotide (DPN) & $0 \cdot 19$ \\
\hline Desamido-DPN & $0 \cdot 14$ \\
\hline Adenine & $0 \cdot 65$ \\
\hline Hypoxanthine & $0 \cdot 62$ \\
\hline \multirow{3}{*}{$\begin{array}{l}\text { U.v.-absorbing spots in extracts from } \\
\text { culture filtrates }\end{array}$} & $0 \cdot 15$ \\
\hline & $\mathbf{0 . 6 1}$ \\
\hline & $0 \cdot 76$ \\
\hline \multirow{5}{*}{$\begin{array}{l}\text { U.v.-absorbing spots in extracts from } \\
\text { culture filtrates after acid hydrolysis }\end{array}$} & $\mathbf{0 \cdot 1 4}$ \\
\hline & 0.24 \\
\hline & $0 \cdot 61$ \\
\hline & $\mathbf{0 \cdot 6 3}$ \\
\hline & 0.77 \\
\hline
\end{tabular}

Effect of adenine and of certain related substances on excretion of nicotinic acid

It is well established that biotin is concerned in the biosynthesis of purines in Saccharomyces cerevisiae (Chamberlain \& Rainbow, 1954; Moat et al. 1956). However, as reported by Chamberlain \& Rainbow (1954), addition of adenine to media containing suboptimal concentrations of biotin brought about only a slight stimulation of growth, although in concentrations above $0.5 \times 10^{-3} \mathrm{M}$-adenine, the organisms did not have the pink colour characteristic of the biotin-deficient yeast. When media containing $0.4 \times 10^{-10} \mathrm{M}$-biotin were supplemented with adenine, excretion of both free and combined nicotinic acid decreased (Fig. 3). Excretion of the free form 
of the vitamin was more sensitive to the presence of adenine, and at $0.7 \times 10^{-3} \mathrm{M}$ adenine, the amount had dropped to less than one-tenth of that excreted in adeninefree medium; increased concentrations of adenine produced no further decrease in the amount of nicotinic acid excreted. The effect on excretion of the combined form of nicotinic acid was somewhat less marked, adenine at $3.5 \times 10^{-3} \mathrm{M}$ being required to produce maximum effect.

However, addition of hypoxanthine or 4-amino-5-imidazole carboxamide, ribotide derivatives of which are concerned in the biosynthesis of purines, had no observable effect, even at $4.0 \times 10^{-3} \mathrm{M}$, on growth of, or nicotinic acid excretion by, the yeast when growing in media containing $0.4 \times 10^{-10} \mathrm{M}$-biotin.

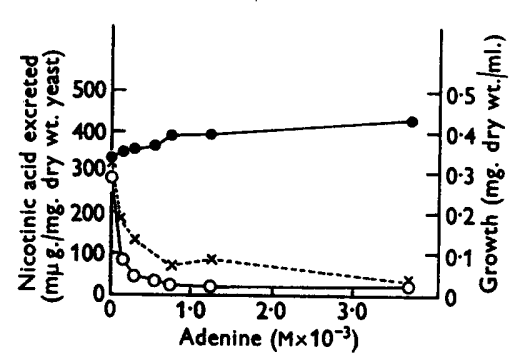

Fig. 8

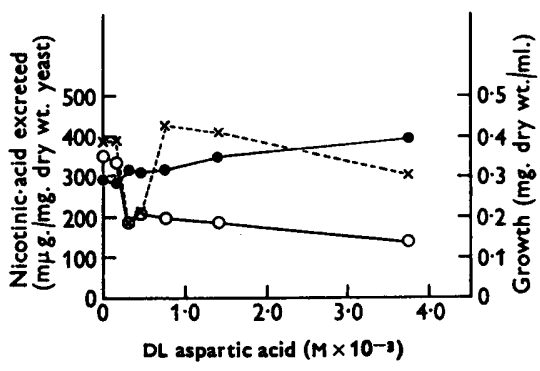

Fig. 4

Fig. 3. Effect of adenine concentration on growth (- - - ) and excretion of free $(\mathrm{O}-\mathrm{O})$ and combined $(x---x)$ nicotinic acid by the yeast in a medium containing $0.4 \times 10^{-10} \mathrm{M}$-biotin after incubation for $181 \mathrm{hr}$.

Fig. 4. Effect of DL-aspartic acid concentration on growth (- -0$)$ and excretion of free $(0-O)$ and combined $(x---x)$ nicotinic acid by the yeast in a medium containing $0.4 \times 10^{-10} \mathrm{M}$-biotin after incubation for $167 \mathrm{hr}$.

Koser, Wright \& Dorfman (1942) reported that aspartic acid was able to replace partially the biotin requirement of the yeast Candida pseudotropicalis; a similar effect was later reported for strains of Saccharomyces cerevisiae (Moat \& Emmons, 1954). The data in Fig. 4 show the effect of DL-aspartic acid on growth and nicotinic acid excretion by the yeast growing in a medium containing $0.4 \times 10^{-10} \mathrm{M}$-biotin. The amino acid had only a slight stimulatory effect on growth of the yeast, although at concentrations above $0.5 \times 10^{-3} \mathrm{M}$, the organisms appeared creamy-white instead of pink. Concentrations of aspartic acid up to $0.5 \times 10^{-3} \mathrm{M}$ caused a decrease in the amounts of free and combined vitamin excreted; but whereas the addition of further aspartic acid had little effect on the amount of free nicotinic acid excreted, the concentration of desamido-DPN in culture filtrates increased sharply, and only decreased when the concentration of amino acid in the medium exceeded $2 \cdot 0 \times 10^{-3} \mathrm{M}$.

\section{DISCUSSION}

Accumulation of biosynthetic intermediates by micro-organisms growing under conditions of metabolic stress has been studied with the object of elucidating the intermediate stages during the biosynthesis of a cell component, and also to discover the mode of action of the metabolic stress. Nicotinic acid occurs in living cells in the form of pyridine nucleotides, DPN and TPN, and accumulation in the culture 
medium of nicotinic acid and desamido-DPN suggests that synthesis of pyridine nucleotides by Saccharomyces cerevisiae is impaired under conditions of biotin deficiency. A deficiency of pyridine nucleotides in the biotin-deficient cells was not demonstrated experimentally in the present work, although this situation has been reported with Piricularia oryzae (Katsuki, 1959a) and with Bacillus macerans (Katsuki, 1959b).

The sequence of reactions involved in the biosynthesis of pyridine nucleotides in several different types of tissue has been studied in various laboratories (Preiss \& Handler, 1958b; Langan, Kaplan \& Shuster, 1959). These studies have indicated that synthesis of DPN proceeds via nicotinic acid, nicotinic acid mononucleotide (desamido-NMN) and desamido-DPN. Accumulation of two of these intermediates namely, nicotinic acid and desamido-DPN, in biotin-deficient cultures of Saccharomyces cerevisiae is in agreement with the finding by Preiss \& Handler (1958b) that this biosynthetic pathway operates in yeast. The absence of nicotinamide from yeast culture filtrates, either free or in nucleotide form, is also significant, for it gives support to the contention that this amide is formed only by degradation of nicotinamide nucleotides, and that free nicotinamide does not participate directly in the synthesis of these nucleotides (Hughes \& Williamson, 1952). Also, since free nicotinic acid and a nicotinic acid nucleotide accumulate under conditions of biotin deficiency, it might be concluded that synthesis of nicotinic acid by the yeast does not require the presence of biotin. In this connexion it may be mentioned that Dalgliesh (1955) reported that in the rat conversion of tryptophan to nicotinic acid was not affected by biotin deficiency.

Accumulation of free nicotinic acid and desamido-DPN in the culture medium under conditions of biotin deficiency suggests that biotin is essential for further metabolism of these intermediates, the vitamin functioning either co-enzymically or in the synthesis of other participants in the reactions. Adenine is required for synthesis of pyridine nucleotides from nicotinic acid, and since biotin is essential for the synthesis of purines in Saccharomyces cerevisiae (Chamberlain \& Rainbow, 1954; Moat, Wilkins \& Friedman, 1956) it might be expected that, under conditions of biotin deficiency, biosynthesis of pyridine nucleotides would be deranged. The marked decrease in excretion of both forms of nicotinic acid that was observed in the present work on adding adenine to the medium suggests that a deficiency of adenine was largely responsible for the impairment in pyridine nucleotide synthesis. This conclusion is further supported by the observation that there was no significant effect on nicotinic acid excretion following addition of hypoxanthine or 4-amino-5imidazole carboxamide, the ribotide derivatives of which are concerned in purine biosynthesis, but which require the presence of biotin for further metabolism (Moat et al. 1956). Adenine was reported to be required in the form of ATP for the conversion of desamido-NMN into desamido-DPN and for the amidation of desamido-DPN (Preiss \& Handler, 1958b). Katsuki (1959a, $b$ ) has reported that Piricularia oryzae and Bacillus macerans, grown in media containing suboptimal concentrations of biotin, contained considerably decreased amounts of ATP as well as of DPN; he concluded that the decreased amounts of DPN were due, not to the absence of DPN-synthesizing enzymes, but to deficiency of ATP. From the difference in the concentrations of adenine required to bring about an equivalent decrease in the excretion of free and combined nicotinic acid by the yeast, it might 
be concluded that the presence of adenine in the medium more easily facilitates the further metabolism of nicotinic acid than the amidation of desamido-DPN. Since ATP is required for both transformations, it is conceivable that the reactions proceed at different sites within the cell, or alternatively, that biotin is also required per se in amidation of desamido-DPN or in the synthesis of glutamine which has been reported to participate in the amidation reaction.

The role of aspartic acid in purine synthesis (Wahba \& Shive, 1954), and in amino group transfer in the purine skeleton (Abrams \& Bentley, 1955) may well explain the biotin-sparing action of this amino acid. From Fig. 4 it can be seen that the effect of aspartic acid on growth of the yeast in biotin-deficient medium was slight, and was, in fact, less marked than previously reported for strains of Saccharomyces cerevisiae (Chamberlain \& Rainbow, 1954; Rose \& Nickerson, 1956) and of other micro-organisms growing under comparable conditions of biotin deficiency; this might, however, be a result of using biotin-deficient yeast cells in the inoculum. But although the effect of the amino acid on growth was slight, excretion of free and combined nicotinic acid was decreased by adding the amino acid up $0.5 \times 10^{-3} \mathrm{M}$. Addition of more aspartic acid had little further effect on excretion of the free form of nicotinic acid; on the other hand, excretion of desamido-DPN was stimulated, although at higher concentrations there was a decrease in the amount which appeared in the growth medium. An explanation of this stimulatory effect is not immediately apparent, although aspartic acid may act indirectly by stimulating synthesis of nicotinic acid. However, even in the presence of $3.75 \times 10^{-3} \mathrm{M}$-aspartic acid, substantial amounts of free and combined nicotinic acid were excreted, which indicates that the biotin requirement in certain of the biotin-dependent reactions concerned in purine synthesis cannot be replaced by aspartate. It is concluded, therefore, that excretion of nicotinic acid and desamido-DPN by the strain of $S$. cerevisiae used in the present work, when growing in media containing suboptimal concentrations of biotin, is a result of the inability of the biotin-deficient yeast to produce adenine, and that this causes a derangement in pyridine nucleotide synthesis, leading to the excretion of the two biosynthetic intermediates.

It is a pleasure to acknowledge the help of Mr R. J. N. Booth and Mr W. R. McAdam during the early stages of this work. The author is also indebted to Dr N. O. Kaplan (Brandeis University, Waltham, Mass., U.S.A.) for a gift of desamido-DPN, and to Miss M. T. Clement (Division of Applied Biology, National Research Council of Canada) for the culture of Saccharomyces cerevisiae.

\section{REFERENCES}

Abrams, R. \& Bentley, M. (1955). Transformation of inosinic acid to adenylic and guanylic acids in a soluble enzyme system. J. Amer. chem. Soc. 77, 4179.

AJl, S. J., Hart, W. R. \& Werkman, C. H. (1950). Biotin in succinic acid oxidation. Enzymologia, 14, 1.

Buss, C. I. (1956). The calculation of microbial assays. Bact. Rev. 20, 243.

Chamberlain, N., Cutss, N. S. \& Rainbow, C. (1952). The formation of pigment and arylamine by yeasts. J. gen. Microbiol. 7, 54 .

Chamberlain, N. \& Rainbow, C. (1954). The formation of diazotisable amine and hypoxanthine by yeast: possible implications in the biosynthesis of purines. J. gen. Microbiol. 11, 180. 
Dalgliesh, C. E. (1955). The relation between thiamine, biotin and tryptophan metabolism studied in the rat. Biochem. J. 61, 328.

Hofmann, K., O'Leary, W. M., Yoho, C. W. \& Liv, T.-H. (1959). Further observations on the lipide stimulation of bacterial growth. J. biol. Chem. 234, 1672.

Hughes, D. E. \& Wimliamson, D. H. (1952). The synthesis of cozymase from nicotinic acid and its derivatives by Lactobacillus arabinosus 17-5. Biochem. J. 51, 330.

KATSUKI, H. (1959a). Studies on the metabolic function of biotin. III. Accumulation of $\alpha$-keto acids in biotin-deficient cultures of Piricularia oryzae. J. Biochem., Tokyo, 46, 621.

KATsUKI, H. (1959b). Studies on the metabolic function of biotin. IV. Function of biotin in $\alpha$-keto acid oxidation. J. Biochem., Tokyo, 46, 979.

Kodicer, E. \& Reddi, K. K. (1951). Paper chromatography of nicotinic acid derivatives. Nature, Lond. 168, 475.

Koser, S. A., Wright, M. H. \& Dorfman, A. (1942). Aspartic acid as a partial substitute for the growth-stimulating effect of biotin on Torula cremoris. Proc. Soc. exp. Biol., N.Y. 51, 204.

Langan, T. A., Kapran, N. O \& Shuster, L. (1959). Formation of the nicotinic acid analogue of diphosphopyridine nucleotide after nicotinamide administration. J. biol. Chem. 234, 2161.

Lardy, H. A., Potter, R. L. \& ElvehJem, C. A. (1947). The role of biotin in bicarbonate utilisation by bacteria. J. biol. Chem. 169, 451 .

Lichstein, H. C. \& Christman, J. F. (1948). The role of biotin and adenylic acid in amino acid deaminases. J. biol. Chem. 175, 649.

Lichstein, H. C. \& UMBreit, W. W. (1947). A function for biotin. J. biol. Chem. 170, 329.

Lones, D. P., Rannbow, C. \& Woodward, J. D. (1958). A diazotisable amine produced by yeast; its chemical nature and factors affecting its accumulation. J. gen. Microbiol. 19, 146.

MoAt, A. G. \& Emmons, E. K. (1954). The amino acid nutrition of yeasts in relationship to biotin deficiency. J. Bact. 68,687 .

Moat, A. G., Wilkins, C. N. \& Friedman, H. (1956). A role for biotin in purine biosynthesis. J. biol. Chem. 223, 985.

Northam, B. E. \& NorRIs, F. W. (1951). Growth requirements of Schizosaccharomyces octosporus, a yeast exacting towards adenine. J. gen. Microbiol. 5, 502.

Preiss, J. \& Handler, P. (1958a). Biosynthesis of diphosphopyridine nucleotide. 1. Identification of intermediates. J. biol. Chem. 233, 488.

Preiss, J. \& Handuer, P. (1958b). Biosynthesis of diphosphopyridine nucleotide. 2. Enzymatic aspects. J. biol. Chem. 233, 493.

Ravel, J. M., Grona, M. L., Humphreys, J. S. \& Shrve, W. (1959). Properties and biotin content of purified preparations of the ornithine-citrulline enzyme of Streptococcus lactis. J. biol. Chem. 234, 1452.

Rose, A. H. (1960). Excretion of nicotinic acid and nicotinic acid adenine dinucleotide by biotin-deficient yeast. Nature, Lond. 186, 139.

Rose, A. H. \& Nickerson, W. J. (1956). Nicotinic acid secretion by biotin-dependent yeasts. J. Bact. 72, 324.

ShIve, W. \& Rogers, L. L. (1947). Involvement of biotin in the biosynthesis of oxalacetic and $\alpha$-ketoglutaric acids. J. biol. Chem. 169, 453.

Singer, T. P. \& Kearney, E. B. (1954). Chemistry, metabolism and scope of action of the pyridine nucleotide coenzymes. Advanc. Enzymol. 15, 79.

Strauss, R. R. \& Moat, A. G. (1958). A role for biotin in yeast glycolysis. J. biol. Chem. $233,765$.

Warba, A. J. \& ShIve, W. (1954). A role of aspartic acid in purine biosynthesis. J. biol. Chem. 211, 155.

WANG, Y. L. \& KodiceK, E. (1943). Vitamin methods. 7. A new modification of the $p$-aminoacetophenone method for estimating nicotinic acid in urine. Biochem. J. 37, 530.

Wruntams, W. L. (1946). Yeast microbiological method for determination of nicotinic acid. J. biol. Chem. 166, 397. 